\title{
Study on modification of calcium carbonate for paper filler
}

\author{
Yanqing $\mathrm{Wu}^{1, \mathrm{a}}$, Chuanshan Zhao ${ }^{1, \mathrm{~b}}$, Yifei Jiang ${ }^{1, \mathrm{c}}$ and Wenjia $\mathrm{Han}^{1, \mathrm{~d}}$ \\ ${ }^{1}$ Key Laboratory of Pulp and Paper Science and Technology of Ministry of Education, QiLu \\ University of Technology, Jinan, Shandong Province,250353, China. \\ ${ }^{2}$ Key Laboratory of Pulp and Paper Science and Technology of Ministry of Education, QiLu \\ University of Technology, Jinan, Shandong Province,250353, China. \\ ${ }^{3}$ Key Laboratory of Pulp and Paper Science and Technology of Ministry of Education, QiLu \\ University of Technology, Jinan, Shandong Province,250353, China. \\ ${ }^{4}$ Key Laboratory of Pulp and Paper Science and Technology of Ministry of Education, QiLu \\ University of Technology, Jinan, Shandong Province,250353, China.

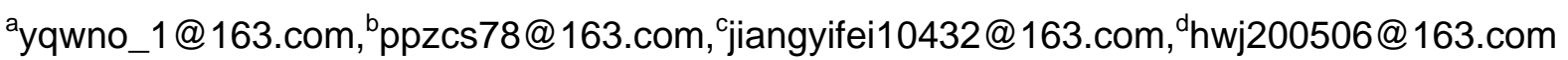

Keywords: GCC, filler modification, physical properties, retention

\begin{abstract}
Using silane coupling agent and ammonium zirconium carbonate to modify heavy calcium carbonate separately. Experiments show that using modified calcium carbonate as a filler made of significantly higher than filling in terms of the physical strength of paper. Under $40{ }^{\circ} \mathrm{SR}$ condition, after $1 \%$ ammonium zirconium carbonate complex of $4 \%$ oxidized starch modified calcium carbonate which works best, to which tensile index up $74.5 \mathrm{~N} . \mathrm{m} / \mathrm{g}$, while the filling GCC is $54.9 \mathrm{~N} \mathrm{.m} \mathrm{/} \mathrm{g.} \mathrm{Through} \mathrm{the} \mathrm{ash} \mathrm{of} \mathrm{calcium} \mathrm{carbonate} \mathrm{filler} \mathrm{finding} \mathrm{that} \mathrm{retention} \mathrm{of} \mathrm{modified} \mathrm{is}$ higher than unmodified ,from $31.35 \%$ to $45.94 \%$.
\end{abstract}

\section{Introduction}

The advantages of calcium carbonate[1] are high brightness[2], fine particles, and improving the opacity of paper significantly. In addition ,fast ink absorption of calcium carbonate, can effectively promote drying of the printing ink, paper with a relatively soft, dense, shiny. The best advantages of calcium carbonate is that can be controlled combustion of the paper, and can effectively improve the air permeability of the paper and the opacity. Therefore, calcium carbonate is the ideal papermaking filler [3].

However, calcium carbonate is an alkaline filler having relatively poor chemical stability, in acidic conditions readily decompose into carbon dioxide gas and produce the bubble, and then the $\mathrm{pH}$ of the slurry is increased, destructs the effect of sizing, and makes the papermaking difficult. Therefore, calcium carbonate as a filler paper, the current multi-use neutral sizing or no sizing of paper and thin printing paper. Calcium carbonate fillers for papermaking --- There are two types of ground calcium carbonate (GCC) and precipitated calcium carbonate (PCC), ground calcium carbonate particle shape is irregular, but the surface is very rough, so it needs to be modified.

Activity of calcium carbonate, also known as modified calcium carbonate[4], surface treated calcium carbonate, calcium abbreviation live, is obtained from the GCC light calcium or surface modified with a surface modifier[5]. The modified calcium carbonate which is small particle size, low oil absorption and good dispersion, more important is having a reinforcement, but also socalled "active", is usually referred to activity of calcium carbonate. The surface modifying agents commonly used of heavy calcium carbonate[6] are mainly coupling agents[7,8], fatty acids and salts, the water-soluble polymer and organic oligomer, etc. There are a silane coupling agent[9], titanate coupling agent and aluminum coupling agent and so on. Fatty acids and their salts are mainly stearate[10], sodium stearate, calcium stearate, zinc stearate, etc. Water-soluble polymers can be divided into natural water-soluble polymers, semisynthetic water-soluble polymers and synthetic water-soluble polymers, Calcium carbonate is used primarily in aqueous systems to improve dispersion stability and compatibility. 
Nowadays the papermaking industry is being plagued by a series of serious environmental problems. Since the world's forest area is gradually narrowing, leading wood fiber pulp prices rising and insufficient supply. What's more, the cost of processing waste generated in the paper process is also significantly improved. Finally, the paper added other energy prices also rose sharply. Therefore, the paper industry is currently faced with two choices, either to pay the high cost or reduce the quantity and quality of the fiber, but will lead to lower quality paper. There is no effect on the strength properties of the paper that the unmodified filler directly into the paper feed system through using the traditional filling method. The strength properties of the paper is often also decline. And the study to improve the negative phenomena is very important. At present, the calcium carbonate filler has become an important development direction of the paper filler. Adding calcium carbonate filled paper, the strength performance is critical, and largely determines the application properties of the paper. In recent years, the international research modified calcium carbonate filler for paper activity, there have been a series of research reports.

\section{Experimental}

\section{Materials and Chemicals}

GCC 、Canadian softwood bleached pulp 、 silane coupling agent A Ammonium zirconium carbonate、Oxidized starch、Neutral dispersant、CPAM

\section{Analytical Methods}

Experimental Method Calcium Carbonate Modified

Selected a modifier : silane coupling agent and zirconium ammonium carbonate modified heavy calcium carbonate, and filling into the pulp, the physical properties of the paper was detected, then determined the final condition.

\section{Pulp processing and Papermaking}

Used Wali beater ease softwood pulp board, then, refining with PFI refiner. Made

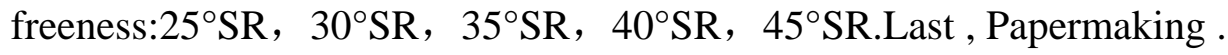

\section{Test results}

Effect of modified calcium carbonate on the sheet properties.

Table1 modifier species

\begin{tabular}{|c|c|c|c|c|c|}
\hline NUM & 1 & 2 & 3 & 4 & 5 \\
\hline Filler & Blank & GCC & GPTMS & $0.1 \% \mathrm{~A}, 2 \% \mathrm{~B}$ & $0.3 \% \mathrm{~A} 2 \% \mathrm{~B}$ \\
\hline NUM & 6 & 7 & 8 & 9 & 10 \\
\hline Filler & $0.5 \% \mathrm{~A} 2 \% \mathrm{~B}$ & $0.7 \% \mathrm{~A} 2 \% \mathrm{~B}$ & $1 \% \mathrm{~A} 2 \% \mathrm{~B}$ & $1 \% \mathrm{~A} 4 \% \mathrm{~B}$ & $1 \% \mathrm{~A} 6 \% \mathrm{~B}$ \\
\hline
\end{tabular}

A: AZC B: oxy-starch

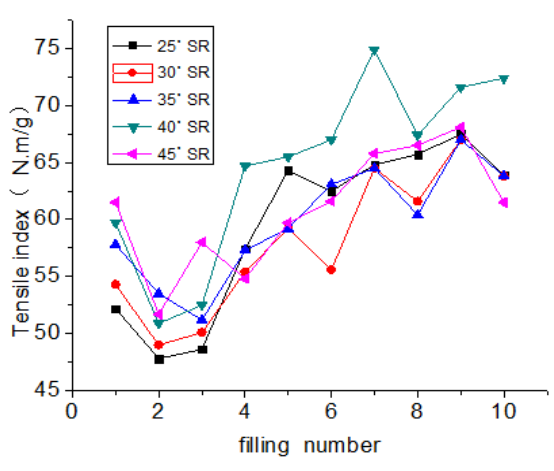

Fig 1 Modifier effects on the tensile index

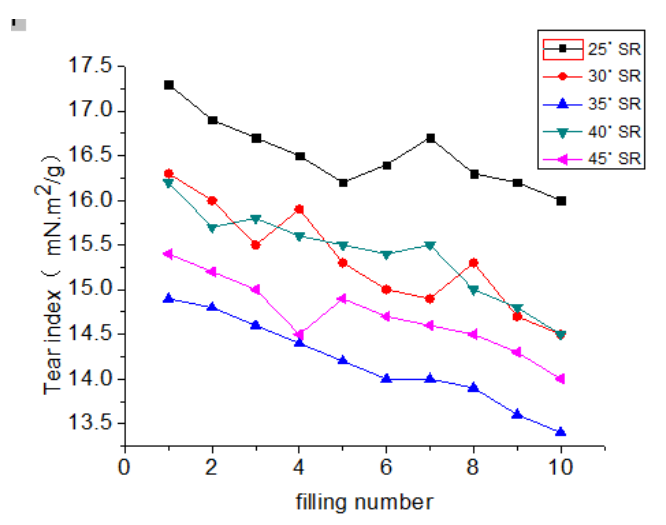

Fig 2 Modifier effects on the tear index 


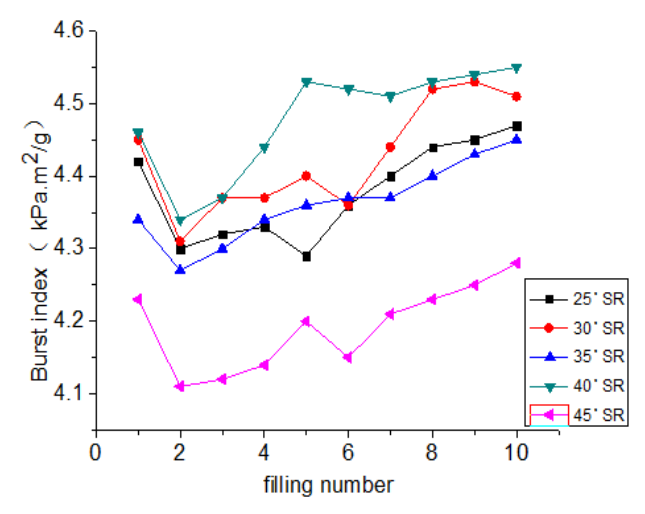

Fig 3 Modifier effects on the burst index

Obtained from the diagram, When the beating degree of $40^{\circ} \mathrm{SR}$, the most comprehensive strength of the paper. This is because the beating makes the fiber surface is deformed, swelling, fibrosis, and cut off a series of fine effect, with the increase of freeness, fiber swelling and thin fibrosis increases, the fiber surface area increases, Free up more hydroxyl groups, to promote hydrogen bonding between the fibers, the bonding strength fiber rising continuously. When it reach a certain freeness, fiber is highly absorbent swelling and fine fibers, it is difficult to further increase the bonding area, the adhesion strength of the fiber has peaked at this time.

\section{Analysis of Filler Retention}

At $40^{\circ}$ SR conditions, compared to the modified calcium carbonate and GCC, the results are shown below:

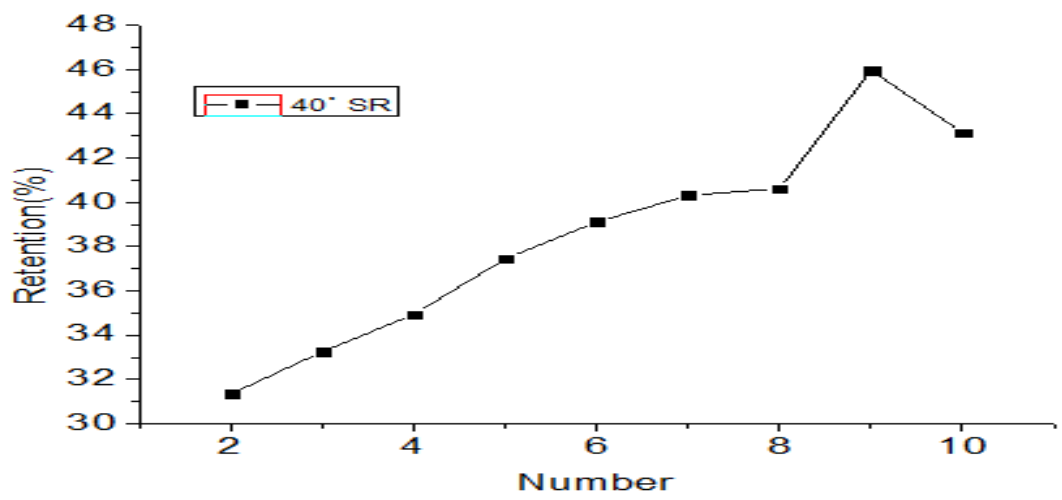

Fig 4 Retention of $40^{\circ}$ SR filler

From chart 4: Under the same conditions freeness, retention of the modified calcium carbonate have a more substantial increase than GCC, It showes that the modified calcium carbonate is a excellent filler. The retention results also illustrate after retention modified filler added enhances paper physical strength. Modified calcium carbonate due to increased retention rates, and thus the physical strength of the sheet less affected thereby improved physical properties. Of which $1 \%$ ammonium zirconium carbonate $4 \%$ starch modified retention rate $45.94 \%$, after the modified silane coupling agent retention rate also increase from 31.35 percent filled not add up to the $33.26 \%$, but the effect is not obvious.

\section{Conclusion}

In the present work, Many experimental results obtained in this experiment, with regard to the study of two modifier of calcium carbonate to calcium carbonate can be effectively modified, but because the silane coupling agent is an oily substance, it is difficult to fully react with the calcium carbonate, which modification of calcium carbonate obviously not as good as ammonium zirconium 
carbonate. The modified ammonium zirconium carbonate is added, paper physical strength was significantly higher than modified silane coupling agent.

At $40^{\circ} \mathrm{SR}$, with $1 \%$ ammonium zirconium carbonate and $4 \%$ starch co-treatment of calcium carbonate, performance of paper strength is the best, the tensile strength and the burst strength are even higher than that applied to fill the paper, the tensile index is $74.9 \mathrm{~N} \cdot \mathrm{m} / \mathrm{g}$, the burst index is $4.58 \mathrm{kPa} \cdot \mathrm{m}^{2} / \mathrm{g}$. This is because under this freeness, the bonding between the fibers maximum. After addition of filler retention and did not increase filled with more substantial increase. Ammonium zirconium carbonate modification made the retention with biggest increase from the original 31.35\% to $31.35 \%$.

\section{References}

[1] Kiyoshi K, Yasunori N, Yashiro O, Kazuto T. Process for preparing calcium carbonate:U. S,6,190,633[P].2001.27-31.

[2] CRITICAL Liu Jing hair, Qu Long stearate of the surface-modified calcium carbonate powder thermal analysis and surface properties change [J] Chemical Research and Application, 1997, 9 (5): 478-481.

[3] Tao Jin, Xianjun Lv, Canming Wang. Application and development of modified calcium carbonateJ]. Shandong University of Science and Technology: Natural Science, 2006,25(2):39-42.

[4] Zhan Cambridge, Yuan Zhilun Fillers with modifiers, paper packing and preparation method and application [P]. Chinese patent 02159775.8,2002-12-25.

[5] Yongzhong Wang, Research and filler surface modification properties of paper [J] paper and paper .2007,26 (6): 54-58.

[6] Jianzhong Chen, Baoping Sun AZG-9301 Development of tiny calcium carbonate heavy activity [J] Anhui Science and Technology, 1995, (8): 13-14.

[7] Yanhua Jiao,The new coupling modified calcium carbonate and its application in PVC [J] Plastics Technology, 2009,37 (11): 83-86.

[8] Yiyan He,Shiguo Du.Application of coupling agent in coatings [J]. Chemical Industry Times, 2002, (02):3-7.

[9] Shiguo Du. Properties and application of silane coupling agent [J]. Hebei Chemical, 1994, (04):35-9.

[10] Robert A,Gill, Bethlehem, Pa. Modified filler material for alkaline Paper and method of use therefore in alkaline Paper making [P].US.5527430,1994-07-21. 